\title{
Genetics and the Veterans Health Administration
}

\author{
Vickie Venne, $\mathrm{MS}^{1}$ and Laurence J. Meyer, PhD, MD'1
}

In this issue of Genetics in Medicine, Dr Maren Scheuner and her team capture the crossroad of the dynamic Veterans Affairs (VA) medical system and expanding clinical genomic services at a critical point in their evolution. They explored the various models of genetic care delivery just as a new service was launched in the Veterans Health Administration, ${ }^{1}$ a rare opportunity to assess implementation. Knowing some of the history of the VA medical system and the strategic plans of the Genomic Medicine Service (GMS) will help put her research findings in context.

In 2008, James B. Peake, then Secretary of Veterans Affairs, established the VA's Genomic Medicine Program. This led to a rapid and ongoing shift in the availability of genomic care. Although Dr Scheuner's research plan and questions were developed long before the survey was launched in November 2011, data collection overlapped with the introduction of a new genetic service in the VA. This new centralized GMS started offering telehealth genetic sessions in June 2011. The data presented by Dr Scheuner were therefore collected just as the GMS was establishing logistics to provide genetic services to veterans - and in only 19 different VA Medical Centers (VAMCs). This is consistent with the survey response that 13 VAMCs obtained genetic consults via telehealth, because some sites may have aligned only after completion of the survey.

However, as of December 2013, the GMS provides genetic services in 51 VAMCs, roughly one-third of all the facilities as shown in Figure 1. The GMS represents a variation of a traditional model of service delivery, in which genetic services are provided using an alternative modality and integrated directly with primary care and other specialties. The veteran can be seen at a VAMC, an outreach clinic, or in certain circumstances, at his or her home via a video encounter. Using the VA's electronic medical record system, documentation of the encounters is shared with the entire care team. Thus, the GMS genetic counselors collaborate with the veteran's healthcare team to provide evidence-based genetic care.

Although the GMS is centralized, with an ultimate goal of ensuring that all veterans have access to quality genetic care, the VA system is not entirely centralized. The Veterans Health Administration oversees the largest integrated health-care delivery system in the United States, but there is more regional variability than most outside the organization appreciate.
The modern VA was formed in the aftermath of World War II. Integral to the creation of the current VA was the affiliation with academic medical centers established by VA Policy Memorandum No. 2, which is still in force. ${ }^{2}$ This policy established that most VA attending physicians are part-timers, with dual appointments at their VAMC and the respective academic affiliate. Of the 153 VAMCs, 124 have academic affiliates.

Dr Scheuner alludes to the Veterans Integrated Service Networks. The Veterans Integrated Service Networks represent a major operational change in the VA that took place in the mid-1990s, under the leadership of Dr Kenneth W. Kizer, then VA Undersecretary for Health. The major impetus for this change was to improve quality of care, a goal that was achieved; by 1997, the VA significantly outperformed the Medicare program on many measures of quality. ${ }^{3}$ The operational impact was a decentralized administration within the VA. Although performance measures were centrally established, the responsibility and resources to accomplish these goals were passed through new regional networks (the Veterans Integrated Service Networks) and out to the medical centers. Associated with this change was an expansion of outpatient care, both within the medical centers and through the establishment of more than 900 freestanding communitybased outpatient clinics. However, the national network was maintained by both updating and linking the VA's electronic health records.

The combination of these two factors-the affiliation with 124 different academic medical centers and the institutionalization of regional and local administrative control with a unified electronic medical record-has led to a significant variation in the local culture of each VAMC. There are differences with respect to the relationship with the academic center, such as the geographic distance and the methods by which subspecialty care at the affiliate can be made available to veterans. There are differences in availability of a particular service within the academic centers as well, clearly evident in a specialty such as genetics. For the 29 VAMCs that are not associated with academic affiliates, access to genetic services is even more limited.

One other historical point is relevant to the availability of genetic care in the VA. Most genetic medicine programs grew within departments of pediatrics and maternal/fetal medicine. 


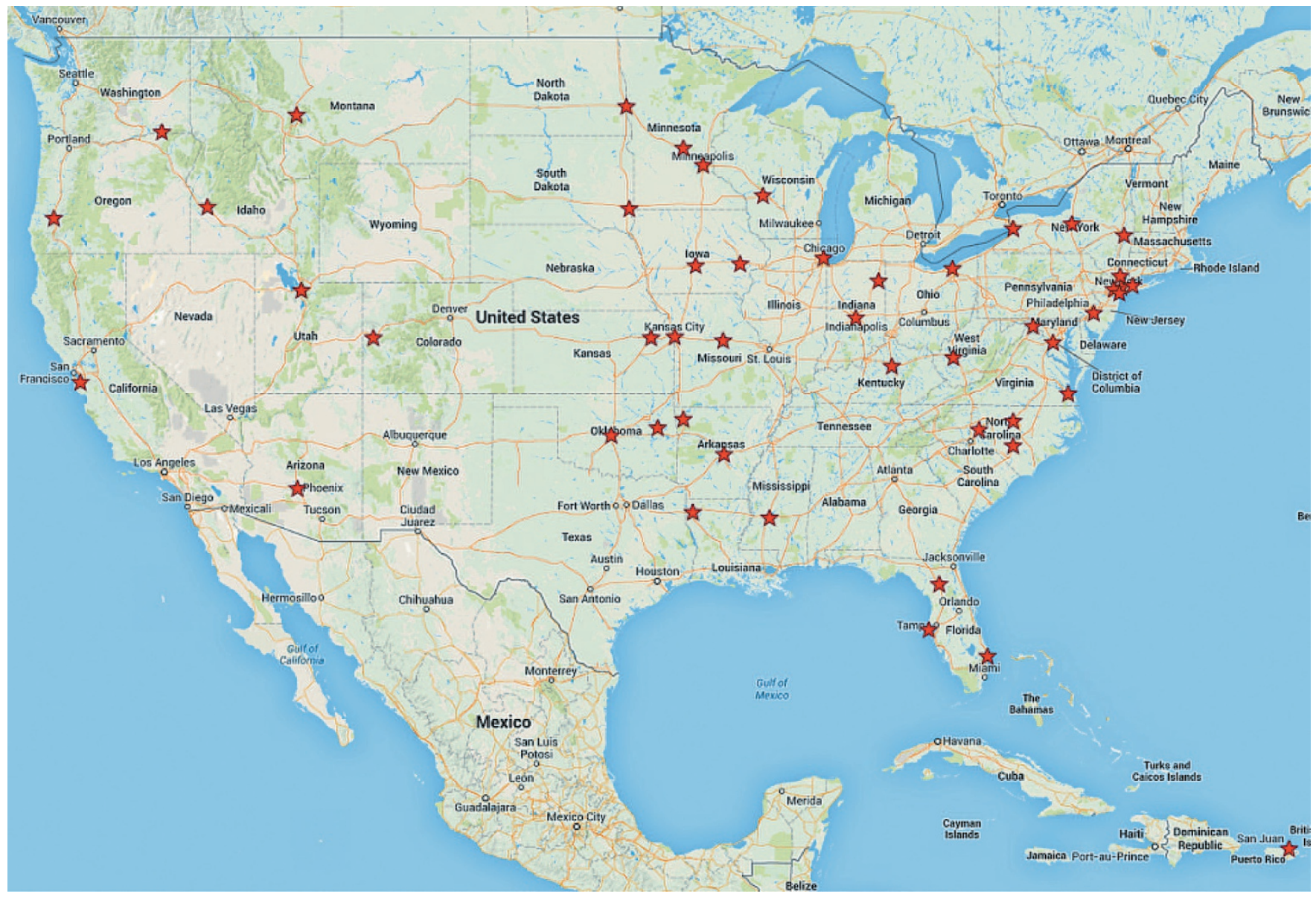

Figure 1 Sites of Veterans Affairs Medical Centers that receive the Genomic Medicine Service telehealth service as of December 2013.

In contrast with the Armed Forces, which offers pediatric services to care for dependents of active-duty servicemen and servicewomen, the VA cares only for adult veterans. Thus, the model for provision of genetic care came under the aegis of each specialty service, such as oncology, neurology, or cardiology.

Finally, since 11 September 2001, the VA's demographic has shifted dramatically. The VA is now emphasizing responses to health concerns of younger men and women who have served since then. In 1988, the VA's Women Veterans Health Program was created to offer medical and psychosocial services to women, who represented only $\sim 4 \%$ of the VA population; in 2014, 10\% of veterans receiving care at VAMCs are female. An example relevant to genetics is that in the majority of facilities, most reproductive care, with the associated genetic aspects, is still referred to a local academic or community hospital.

Dr Scheuner and her colleagues addressed the variability of genetic services available to veterans receiving care at VA facilities. That variability reflects the unique culture of each VAMC based on affiliations with academic medical centers and the decentralized administration. Although still in early stages of implementation, the GMS is poised to provide access to all veterans and consistency in genetic services across all sites while still utilizing the strengths of regional providers and academic affiliates. Strategic plans to incorporate education will increase genetic literacy, so genetic consults with specialists may become more available. Computerized decision support to providers without genetic consults will also undoubtedly expand, especially in areas such as pharmacogenomics.

Because the focus of efforts to date has been developing services with the sites, only modest outreach has been conducted after the new genetic telehealth service is announced at a particular site. This is also consistent with Dr Scheuner's findings, in that some providers in any one facility may not be aware of the availability of the GMS. Frequently, the service providing the impetus to establish GMS telehealth consultation may actively use GMS consultation, and other services may not be uniformly aware of its availability. For example, the women's clinic may use the GMS consultative service, whereas the oncology department refers veterans to the academic affiliate. One can imagine that bringing genetic services into any new facility is a complex process that includes significant logistics and provider education. Currently, in many of these sites, multiple service delivery models continue to offer the best options for veterans to access genomic care. This includes referrals not only to the GMS but also to the academic affiliates. 
Genetic services flourished in pediatric and maternal/fetal clinics, and only in the past few decades have played a significant role in adult genetic care. The VA excelled in providing care to adults and only recently incorporated formalized genetic care into its services. Dr Scheuner's survey provides an interesting perspective regarding access as the GMS is being implemented; it will be fascinating to compare these data in 5 or 10 years to reassess the access to and utilization of genetic care within the VA.

\section{DISCLOSURE}

The authors declare no conflict of interest.

\section{REFERENCES}

1. Scheuner MT, Marshall N, Lanto A, et al. Delivery of clinical genetic consultative services in the Veterans Health Administration. Genet Med 2014; this issue page 609 (gim.2013.202).

2. Policy in association of veterans' hospitals with medical schools. http://www. va.gov/oaa/Archive/PolicyMemo2.pdf. Accessed December 31, 2013.

3. Jha AK, Perlin JB, Kizer KW, Dudley RA. Effect of the transformation of the Veterans Affairs Health Care System on the quality of care. N Engl J Med 2003:348:2218-2227. 